\title{
Performance of Spiked Population Models for Spectrum Sensing
}

\author{
Tan-Thanh Le $\cdot$ Hyung Yun Kong
}

\begin{abstract}
In order to improve sensing performance when the noise variance is not known, this paper considers a so-called blind spectrum sensing technique that is based on eigenvalue models. In this paper, we employed the spiked population models in order to identify the miss detection probability. At first, we try to estimate the unknown noise variance based on the blind measurements at a secondary location. We then investigate the performance of detection, in terms of both theoretical and empirical aspects, after applying this estimated noise variance result. In addition, we study the effects of the number of SUs and the number of samples on the spectrum sensing performance.

Key words: Random Matrix Theory, Cognitive Radio Networks, Spiked Population Model, Spectrum Sensing Techniques, Eigenvalue-Based Spectrum Sensing, Noise Estimation.
\end{abstract}

\section{I . Introduction}

Much recent work has focused on eigenvalue-based spectrum sensing methods for cognitive radio networks (CRNs) [1], [2], where researchers have applied innovations from random matrix theory to calculate the probability of a false alarm as a function of a threshold. This work has also employed many kinds of test statistics, such as the ratio of the largest and the smallest eigenvalues or the ratio of the average and the smallest eigenvalues of the sample covariance matrix. Yonghong and Ying-chang's model in [2] requires a number of samples, $\mathrm{N}$, and a number of secondary users (SUs), K, approaching infinity. A significant gap exists between the simulations and analytical results.

Kortun et al. [1] improved the model by finding an exact threshold and an approximate closed-form performance that agreed well with the empirical results. Independently, Penna et al. [7] derived work similar to [1] and then further analyzed the probability of a miss in [10]. However, the method used in [10] still requires knowledge of noise level and the parameters of primary user (PU) signals and channels, which is impractical in CRN scenarios. To our knowledge, not much research has been carried out regarding the relationship between the threshold and the probability of a miss when using blind spectrum sensing techniques.
This paper considers a novel blind spectrum sensing technique for CRNs, where we analyze a threshold with a constraint on the probability of a miss. CRNs are composed of a specific bandwidth, including multiple PUs and the number of SUs. Based on a variety of research related to the distribution of eigenvalues [3], [4], we apply the results of random matrix theory to the spectrum sensing model. In fact, the practical model is related to a finite number of samples and SUs, while these parameters are infinite in random matrix theory. Therefore, we exploit the limit distribution for approximate results in our applied spectrum sensing model. Furthermore, the observations referred to in this spectrum sensing model undergo interference with by complex Gaussian noise and are affected by fading channels. Performance still depends on the channel parameters, noise powers, and the PU signal powers. However, these parameters are unknown in practice. Therefore, noise estimation schemes must be combined with analytical approaches. This work also focuses on improving the performance of the noise estimation scheme in order to provide an efficient solution to the problem with unknown information.

The rest of this paper is organized as follows. Section 2 presents the sensing model. The analysis of the probability of a miss as a function of threshold is considered in Section 3. Section 4 demonstrates simulation results and discussions. Finally, concluding remarks are given

Manuscript received December 5, 2011 ; Revised February 16, 2012 ; Accepted February 27, 2012. (ID No. 20111205-039J)

Dept. of Electrical. Engineering, University of Ulsan, Ulsan, Korea.

Corresponding Author : Hyung Yun Kong (e-mail : hkong@mail.ulsan.ac.kr)

This is an Open-Access article distributed under the terms of the Creative Commons Attribution Non-Commercial License (http://creativecommons.org/licenses/ by-nc/3.0) which permits unrestricted non-commercial use, distribution, and reproduction in any medium, provided the original work is properly cited. 
in Section 5.

\section{System Model}

The system model can be presented with some assumptions. At the PU side, there are $\mathrm{P}$ sub-bands of interest transmitted by $\mathrm{P}$ entities. Cognitive radio networks have K SUs, which can share their information through high speed transmissions. The high speed requirement for exchange of data is the problem of interest in the model design. Fortunately, these cognitive radio nodes can be linked by wired networks, which help to significantly improve the speed.

The observable signals at the SUs can be expressed as

$$
\mathrm{Y}=\mathrm{HX}+\mathrm{V}
$$

where the $K \times N$ matrix $Y$ is the measurement at the SUs. The $K \times P$ matrix $H$ represents the channels between the PUs and SUs. The $P \times N$ matrix $X$ denotes the transmitted signals from the PUs. In fact, the transmitted signals can be expressed as $X=[x(n)] T, n=1, \cdots, N$, where each $x(n)$ is the $P \times 1$ vector whose elements are obtained from samples of PU signals. Therefore, the covariance matrix of these independent PUs can be written as $\mathrm{E}\left\{\mathrm{xx}^{H}\right\}=\mathrm{C}_{x}=\operatorname{diag}\left(\sigma_{1}^{2}, \ldots, \sigma_{P}^{2}\right)$, where $\sigma_{i}^{2}, \quad i \in[1, P]$ is the variance of the $\mathrm{i}$-th $\mathrm{PU}$ signal. The $\mathrm{K} \times \mathrm{N}$ matrix $\mathrm{V}$ is the complex Gaussian noise with zero mean and variance $\sigma$.

The hypotheses can be expressed as

$$
\begin{aligned}
& \mathcal{H}_{0}:\left.\mathrm{Y}\right|_{\mathcal{H}_{0}}=\mathrm{V}, \\
& \mathcal{H}_{1}:\left.\mathrm{Y}\right|_{\mathcal{H}_{1}}=\mathrm{HX}+\mathrm{V},
\end{aligned}
$$

\section{Performance Analysis}

\section{3-1 Probability of Miss Analysis}

In the case of $\mathcal{H}_{1}$, the measurements at the SUs are rewritten as

$$
Y=\left[\begin{array}{ll}
\widetilde{H} & I_{k}
\end{array}\right]\left[\begin{array}{c}
\tilde{X} \\
V
\end{array}\right]=\left[\frac{1}{\sigma} H C^{1 / 2_{X}} I_{K}\right]\left[\begin{array}{c}
\sigma C^{-1 / 2} X \\
V
\end{array}\right]=J R,
$$

where the covariance matrix of the PU signals are $\mathrm{C}_{x}$

$$
=E\left\{\mathrm{xx}^{\mathrm{H}}\right\}=\operatorname{diag}\left(\sigma_{1}^{2}, \ldots, \sigma_{\mathrm{P}}^{2}\right), \mathrm{J}=\left[\frac{1}{\sigma} \mathrm{HC}_{x}^{1 / 2} \mathrm{I}_{K}\right], \quad \mathrm{R}=\left[\begin{array}{c}
\sigma \mathrm{C}_{x}^{-1 / 2} \mathrm{X} \\
\mathrm{V}
\end{array}\right] .
$$

The test statistic can be found by calculating the eigenvalues $\mathrm{JJ}^{H}=\frac{1}{\sigma^{2}} \mathrm{HC}_{x} \mathrm{H}^{H}+\mathrm{I}_{K}$ as follows:

$$
\begin{aligned}
& \operatorname{det}\left(\mathrm{JJ}^{\mathrm{H}}-\mathrm{I}_{K} t^{(J)}\right)=0, \\
& \Leftrightarrow \operatorname{det}\left(\frac{1}{\sigma^{2}} \mathrm{HC}_{x} \mathrm{H}^{H}+\mathrm{I}_{K}-\mathrm{I}_{K} t^{(J)}\right)=0, \\
& \Leftrightarrow \operatorname{det}\left(\mathrm{HC}_{x} \mathrm{H}^{H}-\sigma^{2}\left(t^{(J)}-1\right) \mathrm{I}_{K}\right)=0, \\
& \Leftrightarrow \operatorname{det}\left(\mathrm{HC}_{x} \mathrm{H}^{H}-\tilde{t} \mathrm{I}_{\mathrm{K}}\right)=0, \tilde{\tau_{i}}=\sigma^{2}\left(t^{(J)}-\right),
\end{aligned}
$$

Using the generalized matrix determinant lemma, we have

$$
\begin{aligned}
& \operatorname{det}\left(C_{x}\right) \operatorname{det}\left(-\tilde{t} I_{k}\right) \operatorname{det}\left(C^{-1_{x}}-\frac{1}{\tilde{t}} H^{H} H\right)=0 \\
& \Leftrightarrow \operatorname{det}\left(C_{x}\right)(-\tilde{t})^{K-P} \operatorname{det}\left(C^{-1_{x}}-\frac{1}{\tilde{t}} H^{H} H\right)=0, \\
& \Leftrightarrow\left\{\begin{array}{c}
\tilde{t}_{i}=0 \quad P+1 \leq i \leq K \\
\operatorname{det}\left(C^{-1_{x}}-\frac{1}{\tilde{t}_{i}} H^{H} H\right)=0,1 \leq i \leq P
\end{array}\right\}
\end{aligned}
$$

Hence,

$$
t_{i}^{(J)}=\frac{\tilde{t_{i}}}{\sigma^{2}}+1
$$

It is easily observed from Eqs. (5) and (6) that the power of each PU and the channel information, represented by $\mathrm{C}_{x}$ and $\mathrm{H}$, respectively, must be known. We first consider the case of known channel state information and unknown PU signal powers. The noise variances must be estimated, and then we can separate into two groups of noises or signals with variances equal to or larger than the estimated one. This technique will be presented and analyzed in Subsection 3.1. Furthermore, SUs do not know channel state information in practice; hence, we approximate $t_{i}^{(J)}$ for a fully blind spectrum sensing model in the Simulation section.

\section{3-2 Methods for Noise Estimation}

In Subsection 3.2, the final expression of miss probability requires knowledge of the sampled covariance matrix of PU signals, the channel matrix, and the noise level. In order to obtain these values, we must estimate the number of PUs and the noise power. Based on previous research [8], we present and analyze the performance of noise estimation, as follows.

The distribution of the largest eigenvalue $\lambda_{1}$ is

$$
\lim _{N, p \rightarrow \infty} \operatorname{Pr}\left\{\frac{\lambda_{1} / \sigma^{2}-\mu(N, p)}{\sigma(N, p)}<x\right\}=F_{W_{2}}(x),
$$

where 


$$
\begin{aligned}
& \mu(N, p)=\frac{1}{N}(\sqrt{N-1 / 2}+\sqrt{p-1 / 2})^{2} \\
& \sigma(N, p)=\frac{1}{N}(\sqrt{N-1 / 2}+\sqrt{p-1 / 2})\left(\frac{1}{\sqrt{N-1 / 2}}+\frac{1}{\sqrt{p-1 / 2}}\right)^{1 / 3},
\end{aligned}
$$

and $F_{W_{2}}(x)$ is the Tracy-Widom distribution with order $2\left(W_{2}\right)[9]$.

In order to determine the total number of active Pus, $\hat{P}$, we apply a series of tests. In other words, we will estimate the noise level of the communication environment at the $p$-th eigenvalue, and then test the likelihood of this $p$-th eigenvalue, $\lambda_{p}$, to determine whether it has arisen from PU signals or noises. In each test, there are two hypotheses, $\mathcal{H}_{0}$ and $\mathcal{H}_{1}$, where the former represents at least $p$ eigenvalues satisfying (9). The latter represents, at most, $(p-1)$ eigenvalues not satisfying the condition in (9), due to the fact that the $p$-th eigenvalue is used as the edges of the two hypotheses, the eigenvalues $\lambda_{i}, i \in[p+1, K]$ are assumed to be arisen from noises.

$\mathcal{H}_{0}$ has at least $p$ eigenvalues satisfying (9).

$\mathcal{H}_{0}$ has at most $(p-1)$ eigenvalues not satisfying (9). The condition used for testing is

$$
\lambda_{p}>\sigma_{P N}^{2}(p)(\mu(N, K-p)+x(b) \sigma(N, K-p)),
$$

where $b=F_{W_{2}}(x)$ is the confidence level, and $x(b)=F_{W_{2}}^{-1}$ (b) is the value determined from inverting the secondorder Tracy Widom distribution. In our work, $b$ is varied from $0.1 \%$ to $0.5 \%$.

The operation can be implemented as described below. If the measurements satisfy hypothesis $\mathcal{H}_{0}$, we increase $p$ and perform the test again. Otherwise, when the hypothesis $\mathcal{H}_{1}$ is satisfied, we stop testing and record the final result $\hat{P}=p-1$. Based on the above operation, $\hat{P}$ can be defined as the solution to the following optimization problem

$$
\hat{P}=\underset{p}{\arg \min }\left\{\begin{array}{l}
\lambda_{p} \leq \sigma_{P N}^{2}(p) \times \\
(\mu(N, K-p)+x(b) \sigma(N, K-p))
\end{array}\right\}-1 .
$$

For Eq. (10), the noise level must be known. Therefore, we next present the method to estimate $\sigma_{P N}^{2}$.

\section{3-2-1 A Simple Method for Noise Estimation}

Equation (1) can be rewritten as

$$
\mathrm{y}=\sum_{j=1}^{P} \mathrm{~h}_{j} \mathrm{x}_{j}+\sigma \xi
$$

The covariance matrix can be modified to a diagonal matrix as

$$
U^{T} C_{y} U=\left[\begin{array}{ll}
A_{11} & \\
& A_{22}
\end{array}\right]+\sigma^{2} I_{K},
$$

where $\mathrm{U}$ is now an unknown $K \times K$ matrix whose columns are the eigenvectors of $\mathrm{C}_{y}, A_{11}=\left[\begin{array}{cc}\lambda_{1} & \times \\ & \ddots \\ \times & \lambda_{2}\end{array}\right]$, and $\mathrm{A}_{22}=0(K-P)$. It is clear that, with a very large number of samples $(N \rightarrow \infty)$, the first $P$ eigenvalues of the covariance matrix are $\lambda_{i}+\sigma^{2}, i=1, \cdots, P$, and the remaining $(K-P)$ eigenvalues are $\sigma^{2}$. The eigenvalues are separated into two groups, the group of signals with estimated eigenvalues larger than $\hat{\sigma}^{2}$ and the group of noises with the remaining eigenvalues. Unfortunately, the noise eigenvalues are widely spread in the case of a finite number of samples. In this setting, we define the covariance matrix for a finite number of samples and observe that

$$
\mathrm{C}_{y N}=\sum_{j=1}^{N} \mathrm{y}_{j} \mathrm{y}_{j}^{H} .
$$

In practice, the sampled covariance matrix can be written as

$$
\mathrm{U}^{T} \mathrm{C}_{y N} \mathrm{U}=\left(\begin{array}{ccc}
\tau_{1} & & \times \\
& \ddots & \\
\times & & \tau_{K}
\end{array}\right)+\left(\begin{array}{lll}
0 & & \times \\
& \ddots & \\
\times & & 0
\end{array}\right)
$$

where $\tau_{i}=\frac{1}{N} \sum_{j=1}^{N}\left(y_{i} u_{i}\right)^{2}$. It is easily seen that the diagonal elements from $(P+1)$ to $K$ are due to noise. By averaging these elements, we derive the noise variance as

$$
\begin{aligned}
\sigma^{2} & =\frac{1}{K-P} \sum_{i=P+1}^{K} \tau_{i}=\frac{1}{K-P}\left(\sum_{i=1}^{K} \lambda_{i}-\sum_{i=1}^{P} \tau_{i}\right) \\
& =\frac{1}{K-P}\left(\sum_{i=P+1}^{K} \lambda_{i}+\sum_{i=1}^{P}\left(\lambda_{i}-\tau_{i}\right)\right)
\end{aligned}
$$

However, the matrix $U$ is unknown. Hence, we must estimate $\tau_{i}$. In the simple form, we set $\tau_{i}=\lambda_{i}$ and get

$$
\hat{\sigma}_{\text {simple }}^{2}=\frac{1}{K-P}\left(\sum_{i=P+1}^{K} \lambda_{i}\right)
$$

\section{3-2-2 Improvement in Noise Estimation}

In order to improve the estimated noise variance, we now diagonalize the upper left submatrix $\mathrm{U}_{1}^{T} \mathrm{C}_{y \mathrm{~N}} \mathrm{U}_{1}$, as 
in $[8]$

$$
\mathrm{U}_{1}^{T} \mathrm{C}_{y N} \mathrm{U}_{1}=\left(\begin{array}{ll}
\mathrm{B}_{11} & \mathrm{~B}_{12} \\
\mathrm{~B}_{21} & \mathrm{~B}_{22}
\end{array}\right)
$$

where $\left(\begin{array}{lll}\phi_{1} & & 0 \\ & \ddots & \\ 0 & & \phi_{P}\end{array}\right), \mathrm{B}_{12}=\mathrm{B}_{21}^{T}=Z$ and $\mathrm{B}_{22}=\left(\begin{array}{ccc}\tau_{P+1} & & 0 \\ & \ddots & \\ 0 & & \tau_{K}\end{array}\right)$.

The eigenvalues of $\mathrm{U}_{1}^{T} \mathrm{C}_{y \mathrm{~N}} \mathrm{U}_{1}$ are

$$
\lambda_{j} \approx \Phi_{j}+\sum_{i=P+1}^{K} \frac{Z^{2_{i j}}}{\Phi_{j}-\tau_{i}}, j \in[I, P],
$$

where $z_{i j}^{2} \approx E\left\{z_{i j}^{2}\right\}=\frac{1}{N} \phi_{j} \sigma^{2}$. Therefore, Eq. (18) can be derived as

$$
\lambda_{j} \approx \phi_{j}+\frac{1}{N} \sigma^{2} \sum_{i=P+1}^{K} \frac{\phi_{j}}{\phi_{j}-\sigma^{2}}=\phi_{j}+\frac{K-P}{N} \sigma^{2} \frac{\phi_{j}}{\phi_{j}-\sigma^{2}},
$$

where $j \in[1, P]$. To this end, we iterate Eqs. (15) and (19) to estimate the noise variance

$$
\left\{\begin{array}{l}
\hat{\sigma}_{i m}^{2}=\frac{1}{K-P}\left(\sum_{i=P+1}^{K} \lambda_{i}+\sum_{i=1}^{P}\left(\lambda_{i}-\hat{\phi}_{i}\right)\right) \\
\hat{\phi}_{i}^{2}-\hat{\phi}_{i}\left(\lambda_{\mathrm{i}}+\hat{\sigma}_{i m}^{2}\left(1-\frac{K-P}{N}\right)\right)+\hat{\lambda}_{i} \hat{\sigma}_{i m}^{2}=0
\end{array}\right.
$$

with the initial value $\hat{\sigma}_{0}^{2}=\hat{\sigma}_{\text {simple }}^{2}\left(1-\frac{P}{N}\right)$ for better performance [5].

After estimating $\hat{\sigma}_{i m}^{2}$, which is used for missed probability analysis with known channel state information and PU signal powers, we also use $\lambda_{i}, i \in[I, P]$ to calculate the eigenvalue $t_{i}^{(J)}$ as in Section 4 for the case of blind spectrum sensing models.

\section{3-3 The Probability of Miss}

Based on the estimated result of noise variance, $\hat{\sigma}_{i m}^{2}$, we perform the calculation of $P_{m}$. Note that the following expressions use $\sigma^{2}$ instead of $\hat{\sigma}_{i m}^{2}$ From [6], we have the following theorem with

$$
\begin{aligned}
& \mu\left(t_{1}^{(J)}\right)=\sigma^{2} t_{1}^{(J)}\left(1+\frac{c}{t_{1}^{(J)}-1}\right) \\
& \sigma\left(t_{1}^{(J)}\right)=\sigma^{2} t_{1}^{(J)} \sqrt{1-\frac{c}{\left(t_{1}^{(J)}-1\right)^{2}}}
\end{aligned}
$$

where $c=K / N$.

\section{3-3-1 Theorem 1}

Ref. [3] Consider the sequence of a complex Wishart matrix with $\mathrm{C} x$. Let $1 \leq m \leq P$, we have the case of identifiable signals; i.e., the spiked eigenvalues are above the critical limit $1+c^{1 / 2}$. If $t_{1}^{(J)}=\ldots=t_{m}^{(J)}>1+c^{1 / 2}$. Let

$$
\varepsilon_{1}=\frac{\sqrt{N}}{\sigma\left(t_{1}^{(J)}\right)}\left(\hat{\lambda}_{1}-\mu\left(t_{1}^{(J)}\right)\right)
$$

then

$$
\lim _{N . P^{T \mathrm{M}} \infty} \operatorname{Pr}\left(\varepsilon_{1} \leq \gamma\right)=\mathcal{G}_{m}(\gamma),
$$

where $\mathcal{G}_{m}(\gamma)$ is the finite Gaussian unitary ensemble (GUE) distribution given as

$F_{G U E_{m}}(\gamma)=(2 \pi)^{-\frac{m}{2}}\left(\prod_{k=1}^{m} k !\right)^{-1} \times$
$\int_{-\infty}^{\gamma} \cdots \int_{-\infty 1 \leq i<j \leq m}^{\gamma} \prod_{i}-\left.\xi_{j}\right|^{2} \prod_{j=1}^{m} \exp \left(-\frac{1}{2 \xi_{j}^{2}}\right) d \xi_{1} \cdots d \xi_{m}$

Based on Eq. (24), the distribution of the largest eigenvalue is $\lim _{N, P \rightarrow \infty} f \varepsilon_{1}(x)=f_{g m}(x)$.

In order to determine the distribution of the smallest eigenvalues, which are the remaining $(K-P)$ eigenvalues, we adhere to Theorem 2 [4]. Let

$$
\begin{aligned}
& \tilde{c}=\frac{K-P}{N} \\
& \mu(\tilde{\mathrm{c}})=\sigma^{2}\left(\tilde{\mathrm{c}}^{1 / 2}-1\right)^{2}, \\
& \sigma(\tilde{\mathrm{c}})=\sigma^{2}\left(\tilde{\mathrm{c}}^{1 / 2}-1\right)\left(\tilde{\mathrm{c}}^{-1 / 2}-1\right)^{1 / 3},
\end{aligned}
$$

\section{3-3-2 Theorem 2}

Consider the sequence of a complex Wishart matrix $\tilde{\mathrm{C}}_{x}$ with size $(K-P) \times(K-P)$ (Note that $\tilde{\mathrm{C}}_{x}$ is the lower right submatrix of $\left.\mathrm{C}_{x}\right) . \lambda_{K}$ is the smallest eigenvalue of $\tilde{\mathrm{C}}_{x}$. The variable $\varepsilon_{K}=\frac{N^{2 / 3}}{\sigma(\tilde{c})}\left(\hat{\lambda}_{K}-\mu(\tilde{c})\right)$ will be satisfied

$$
\lim _{N,(K-P)^{T M} \infty} \operatorname{Pr}\left(\varepsilon_{K} \leq \gamma\right)=F_{W_{2}}(\gamma) .
$$

Based on Eq. (29), the distribution of the smallest eigenvalue is $\lim _{N, P \rightarrow \infty} f \varepsilon_{K}(x)=F_{W_{2}}(x)$.

Now we can establish the ratio test as follows, 


$$
T=\frac{\hat{\lambda}_{1}}{\hat{\lambda}_{K}}=\frac{N^{-1 / 2} \varepsilon_{1 \sigma}\left(t_{1}^{(J)}\right)+\mu\left(t_{1}^{(J)}\right)}{N^{-2 / 3} \varepsilon_{K} \sigma(\tilde{c})+\mu(\tilde{c})} .
$$

Following [7], the joint distribution of the largest and smallest eigenvalues is

$$
f_{T \mid H_{1}}(t)=\int_{0}^{\infty} x f_{\lambda_{1}}(t x) f_{\lambda_{K}}(x) d x,
$$

where the distributions of the largest and smallest eigenvalues are derived from $f_{\varepsilon_{1}}(x)$ and $f_{\varepsilon_{K}}(x)$, respectively, and are given as

$$
\begin{aligned}
& f_{\lambda_{1}}(x)=\frac{N^{1 / 2}}{\sigma\left(t^{(J)}\right)} f_{G_{m}}\left(\frac{N^{1 / 2}}{\sigma\left(t^{(J)}\right)}\left(x-\mu\left(t^{(J)}\right)\right)\right), \\
& f_{\lambda_{K}}(x)=\frac{N^{2 / 3}}{|\sigma(\tilde{c})|} f_{w_{2}}\left(\frac{N^{\frac{2}{3}}}{\mid \sigma\left(\begin{array}{c}
c \\
c
\end{array}\right)}\left(x-\mu\left(\begin{array}{l}
\tilde{c}) \\
\mid c
\end{array}\right)\right) .\right.
\end{aligned}
$$

Therefore, the probability of a miss can be calculated as

$$
P_{m}=\operatorname{Pr}\left(T<\gamma \mid H_{1}\right)=F_{T \mid H_{1}}(\gamma)
$$

and the threshold is the inversion of Eq. (34)

$$
\gamma\left(P_{m}\right)=F_{T \mid H_{1}}^{-1}\left(P_{m}\right)
$$

It is easily observed that the threshold derived from Eq. (35) depends on the channel matrix and the PU signal powers. This is due to the fact that $F_{T \mid H_{1}}(\gamma)$, which includes $\mathrm{H}$ and $\mathrm{C} x$. Information on the PU signal powers is included in Subsection 3.1, and the model considered here is a CSI case. In the next section, we approximate $t_{1}^{(J)}$ in order to obtain the results in the case of the fully blind spectrum sensing model.

\section{Simulation Results and Discussions}

After noise estimation, the results are the noise level $\sigma_{P N}^{2}$ and the number of PU signals, $P$ est, with the probability of the correct number of PU signals $\operatorname{Pr}\left(P_{\text {est }}=\mathrm{P}\right)$. The covariance matrix of the measurements at $K$ of the SUs can then be diagonalized as in Eq. (14) and the first $P$ eigenvalues $\hat{\lambda}_{i}, i \in[1, P]$ represented as $P$ PU signal powers are approximated as Eq. (19). Substituting these eigenvalues to Eq. (7) at $i=1$, we have

$$
t_{1}^{(J)}=\frac{\tilde{t_{1}}}{\sigma^{2}}+1 \approx \frac{\hat{\lambda_{1}}-\sigma_{P N}^{2}}{\sigma_{P N}^{2}}+1=\widehat{\lambda_{1}} .
$$

Proof: When $N$ is very large, the approximation is as follows:

$$
C_{y N} \approx \mathrm{HC}_{x} \mathrm{H}^{H}+C_{v},
$$

where $\mathrm{C}_{v}$ is the sampled covariance matrix of noise. From [11], Weyl's inequality theorem reads as

$$
\tilde{t_{p}}+\lambda_{\max }\left(\mathrm{C}_{v}\right) \leq \hat{\lambda}_{1} \leq \tilde{t}_{1}+\lambda_{\max }\left(\mathrm{C}_{v}\right)
$$

where $\lambda_{\max }\left(\mathrm{C}_{v}\right)$ is the estimated noise eigenvalue, $\sigma_{P N}^{2}$, which is calculated as in Subsection $3.1, \tilde{t}_{1}, \tilde{t}_{2}, \ldots, \tilde{t}_{P}$ are $P$ eigenvalues of $\mathrm{HC}_{x} \mathrm{H}^{H}$, and $\hat{\lambda}_{1}$ is the largest eigenvalue of $\mathrm{C}_{y N}$. Moreover, at very large $N$, the simulation shows that the following approximation performs in a manner that is in good agreement with the empirical results

$$
\hat{\lambda}_{1} \approx \tilde{t}_{1}+\lambda_{\max }\left(\mathrm{C}_{v}\right)=\tilde{t_{1}}+\sigma_{P N}^{2}
$$

Substituting Eq. (39) into Eq. (36), we have the final result.

We now perform spectrum sensing without using any information from the channels or the noise level, in this case. We note that noise estimation relates to the correct number of $\operatorname{PUs} \operatorname{Pr}\left(P_{\text {est }}=P\right)$, so the final probability of a miss is $\hat{P}_{m}=P m \times \operatorname{Pr}\left(P_{\text {est }}=P\right)$.

Now, we analyze the effects of parameters such as $K$, $N$, and $P$ on the noise estimation performance and therefore the spectrum sensing performance. In Fig. 1, the miss probabilities for the unknown model, the ideal model (known model), and the empirical simulation are calculated using various numbers of samples and SUs. As illustrated in this figure, the gap between the ideal and the unknown schemes is slightly too small. In particular, when the number of SUs and samples are increased $((N, K)=(2,000,40),(N, K)=(2,000,50)$ and $(N$, $K)=(800,40))$, the results of both cases are the same. To confirm a good match between the theoretical analysis and the empirical experiment, the model uses only $N=$ 2,000 or $N=800$ samples, which are appropriate for the practical model.

In order to completely understand the estimation scheme, Fig. 2 shows the performance when estimating PU signal powers. Even though a pair of samples and the number of SUs $(N, K)$ are chosen to be $(1,000,50)$ (i.e., a not very large $N)$, the estimated power densities agree well with the original power densities. A larger number of samples gives a more exact result for the estimate of the powers. Hence, if we want to obtain a more precise estimate in order to reduce the difference between the theoretical and empirical results, we slightly increase the number of samples $N$. 


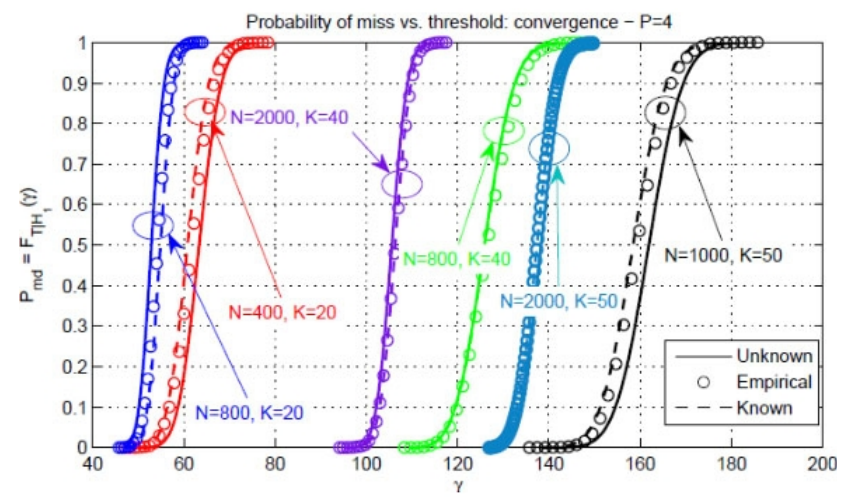

Fig. 1. Probability of miss vs. the threshold in the case of $P=4$ and variable $K$ and $N$.

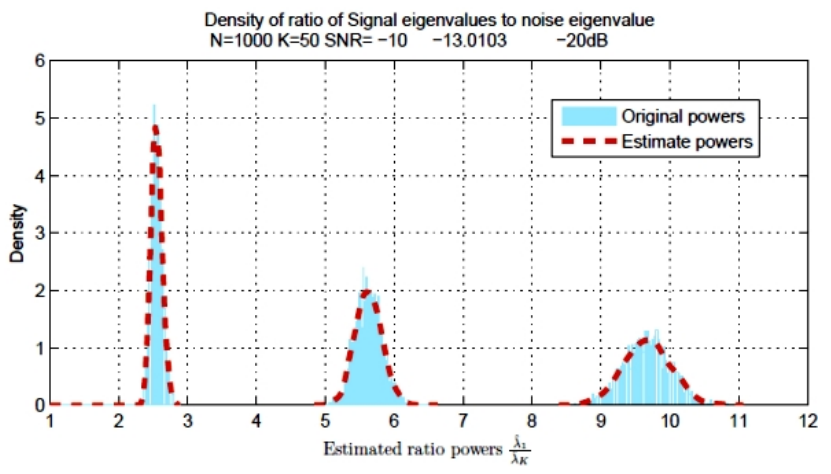

Fig. 2. Density of the ratio of signal eigenvalues to noise eigenvalues in the case of $P=3, K=50$ and $N=1,000$.

Fig. 3 shows the receiver operating characteristic (ROC), which describes the sensitivity of the spectrum sensing model. We also note that, in the case of $H_{0}$, the probability of a false alarm, $P_{f a}$, is calculated based on the results in [7], which include the analysis for $P_{f a}$ as a function of a decision threshold.

Fig. 4 shows the convergence of our proposed scheme at $P=4$ with the constraint $c=0.1$. It is clearly observed that the increases in both $\mathrm{K}$ and $\mathrm{N}$ confirm the convergence of the curve represented by the relation between $P_{m}$ and the threshold. In the case of $N=80$ and $K=8$, the estimated scheme no longer agrees with the known scheme due to the fact that noise estimation performance is heavily degraded when $N$ is too low. Moreover, after the noise estimation stage, the performance of the spectrum sensing technique is also affected by very low $K$ and $N$. Increases in both $N$ and $K$ with the constraint $N / K=c$ ensure that the performance of the analytical unknown case matches well with that of the empirical known case.

Finally, we did not refer to the energy detection technique (ED) in our work; our focus was on comparison of our proposed method with state-of-the-art eigenvaluebased approaches. Several other studies [1], [2], [7],

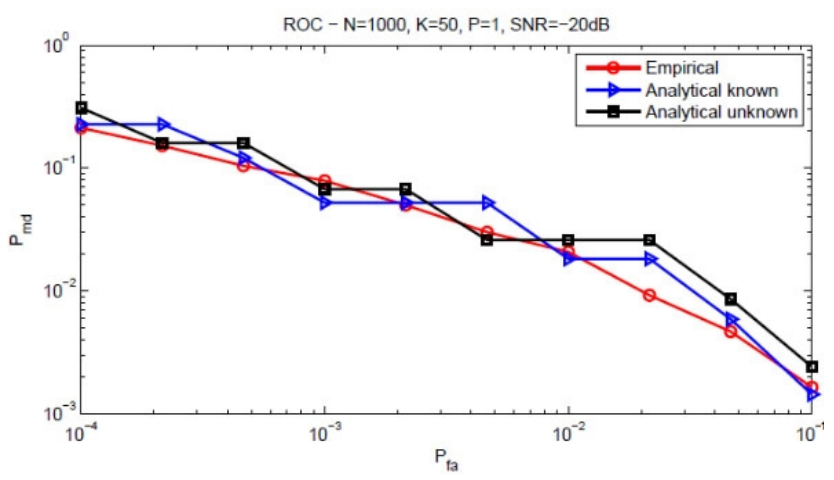

Fig. 3. Probability of miss vs. probability of a false alarm in the case of $P=1, K=50$ and $N=1,000$.

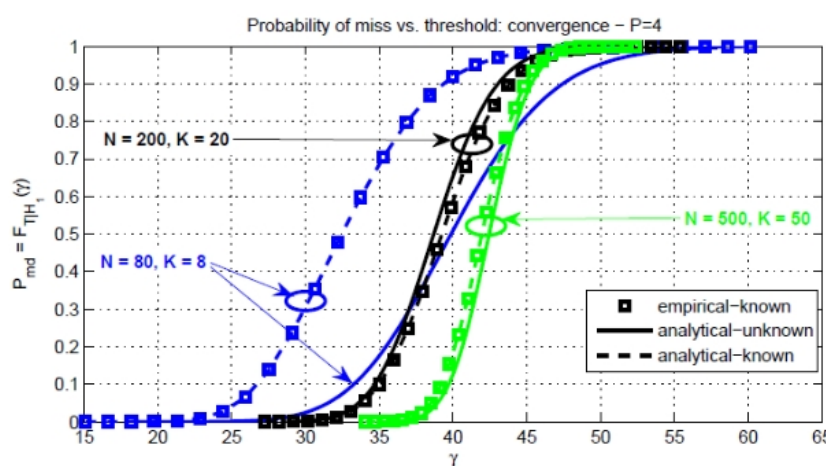

Fig. 4. Miss probability vs. threshold in the case of $P=4$ and variable $K$ and $N$ with the constraint $c=K / N=0.1$.

[10] have confirmed that the eigenvalue-based methods outperform the ED.

\section{Conclusion}

Our work considered a new sensing model for cognitive radio networks, where multiple SUs blindly sense the band of interest, including multiple PUs. A novel analysis is presented to determine the threshold based on the probability of a miss, which is a new method that utilizes the best knowledge from spectrum sensing. Moreover, the proposed hybrid analytical simulation method enhances the blind features of spectrum sensing models since the unknown noise level, and hence the parameters of PU signals and channels, are estimated with small errors. We also show the effects of the estimation scheme and attempt to improve its performance. As a consequence, the empirical simulation results illustrate small differences between the performances of the ideal (known information) and estimate (blind) models.

\section{References}

[1] A. Kortun, T. Ratnarajah, M. Sellathurai, and C. Zhong, "On the performance of eigenvalue-based spectrum sensing for cognitive radio," in IEEE DySPAN 2010, 
2010.

[2] Z. Yonghong, L. Ying-chang, "Eigenvalue-based spectrum sensing algorithms for cognitive radio," IEEE Transactions on Communications, vol. 57, no. 6, pp. 1784-1793, 2009.

[3] S. Peche, "Universality results for the largest eigenvalues of some sample covariance matrix ensembles," Probability Theory and Related Fields, vol. 143, no. 3, pp. 481-516, 2009.

[4] O. Feldheim, S. Sodin, "A universality result for the smallest eigenvalues of certain sample covariance matrices," Geometric and Functional Analysis, vol. 20, no. 1, pp. 88-123, 2010.

[5] N. M. Faber, L. M. C. Buydens, and G. Kateman, "Aspects of pseudorank estimation methods based on the eigenvalues of principal component analysis of random matrices," Chemometrics and Intelligent Laboratory Systems, vol. 25, no. 2, pp. 203-226, 1994.

[6] D. Feral, S. Peche, "The largest eigenvalues of sam-

\section{Tan-Thanh Le}

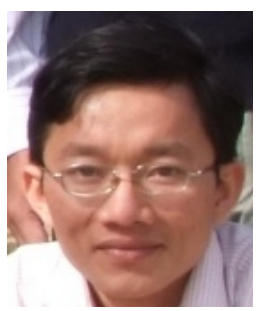

received the B.S. degree in Telecommunication Engineering from Poly-technique University of Hochiminh, Vietnam in 2002. In 2005, he got the degree of Master from Poly-technique University of Hochiminh, Vietnam in major of Electrical and Electronics Engineering. Since 2009, he has been studying Ph.D. program at University of Ulsan, Korea. His major research area is Cognitive Radio Network, Cooperative Communication. ple covariance matrices for a spiked population: Diagonal case," Journal of Mathematical Physics, vol. 50, no. 7, pp. 073302-073333, 2009.

[7] F. Penna, R. Garello, and M. A. Spirito, "Cooperative spectrum sensing based on the limiting eigenvalue ratio distribution in Wishart matrices," IEEE Communications Letters, vol. 13, no. 7, pp. 507-509, 2009.

[8] S. Kritchman, B. Nadler, "Non-parametric detection of the number of signals: Hypothesis testing and random matrix theory," IEEE Transactions on Signal Processing, vol. 57, no. 10, pp. 3930-3941, 2009.

[9] I. M. Johnstone, "On the distribution of the largest eigenvalue in principal components analysis," $\mathrm{An}$ nals of Statistics, vol. 29, 2001.

[10] F. Penna, R. Garello, "Theoretical performance analysis of eigenvalue-based detection," in Preprint, 2009.

[11] R. Bhatia, Matrix Analysis, Springer, 1997.

\section{Hyung Yun Kong}

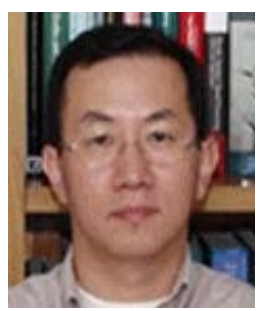

received the M.E. and Ph.D. degrees in electrical engineering from Polytechnic University, Brooklyn, New York, USA, in 1991 and 1996, respectively, He received a BE in electrical engineering from New York Institute of Technology, New York, in 1989. Since 1996, he has been with LG Electronics Co., Ltd., in the multimedia research lab developing PCS mobile phone systems, and from 1997 the LG chairman's office planning future satellite communication systems. Currently he is a Professor in electrical engineering at the University of Ulsan, Korea. His research area includes channel coding, detection and estimation, cooperative communications, cognitive radio and sensor networks. He is a member of IEEK, KICS, KIPS, IEEE, and IEICE. 